hearts. Whether these distinct characteristics are altered differently by an ischaemic insult, thus explaining variations in vulnerability, is not presently known. This is currently under investigation.

\section{P14 MITOCHONDRIAL REGULATION OF EXOSOMAL MICRORNA CARGO MEDIATES CELL PROLIFERATION IN SYNTHETIC VASCULAR SMOOTH MUSCLE CELLS}

P Coats, Z Al-Sulti. Strathclyde Institute of Pharmacy and Biomedical Sciences, University of Strathclyde, Glasgow, UK

\subsection{6/heartjnl-2018-BSCR.19}

In this work we aimed to study potential correlation between the synthetic hyperproliferative vascular smooth muscle (VSM) cell and exosomal-dependent signalling.

We have previously shown mitochondrial bioenergetics are upregulated in synthetic hyperproliferative VSM cells.

Total exosomal release was increased in synthetic VSM cells vs wild type (WT) cells. Total protein and RNA within exosomes in synthetic VSM was significantly greater when compared with WT VSM cells. Addition of exosomes isolated from WT or synthetic hyperproliferative cells to VSM cell cultures resulted in $4.7 \% \pm 2.3 \%$ and $23.4 \% \pm 4.6 \%$ respectively, $\mathrm{p}<0.05$. qRT-PCR of exosomal contents (synthetic VSM cell vs WT VSM cells) highlighted a significant reduction in proapoptotic genes (Bnip3, SOD1, SOD2), a reduction in significant tumour suppressor genes (Pmaip1, p53) and significant reduction in cell cycle regulator Cdkn2a. Likewise, RNA for PI3K, 4EBP1 and mTOR were all significantly greater in exosomes isolated from synthetic VSM cell vs WT VSM cells. miRNAome sequencing highlighted significant differences in exosome contents. Notably a 10 -fold increase in pro-mitogenic/synthetic miR21 and 3.5-fold loss of anti-mitogenic/synthetic miR145, synthetic VSM cell vs WT VSM cells. The use of selective anti-miRs/miR mimetics in cell proliferation assays confirmed that both miR145/miR21 are regulators of VSM cell phenotype and proliferation.

Inhibition of mitochondrial bioenergetics or mitochondrial dynamics restored the exosomal yield, exosomal contents, RNA and miRNA similar to that measured in WT cells.

Our results implicates exosomes and their miRNA contents as crucial mediators of cellular proliferation in synthetic VSM cells

\section{P15 CYCLOSPORINE A IS PROTECTIVE AGAINST OXIDATIVE STRESS IN ADULT BUT NOT IN IMMATURE ISOLATED CARDIOMYOCYTES}

\footnotetext{
${ }^{1,2} \mathrm{MJ}$ Lewis, ${ }^{1,2} \mathrm{~J} \mathrm{Hall}{ }^{*},{ }^{1,2} \mathrm{~L}$ Keylock*${ }^{*}{ }^{1,2} \mathrm{H}$ Lin, ${ }^{1,2} \mathrm{~K}$ Summers, ${ }^{1,2} \mathrm{MS}$ Suleiman. ${ }^{*}$ These authors contributed equally to the project; ' School of Clinical Sciences, University of Bristol; ${ }^{2}$ Bristol Heart Institute, Bristol Royal Infirmary, Upper Maudlin St, Bristol
}

\subsection{6/heartjnl-2018-BSCR.20}

The vulnerability of intact rat hearts to ischaemia and reperfusion injury changes during post-natal development with 14 days old being most resistant. The mechanism for this is multifactorial, but one significant element is the changing characteristics of the mitochondrial permeability transition pore (MPTP). We investigated the effect of oxidative stress and $\mathrm{Ca} 2+$ loading on cardiomyocyte viability in cell suspension throughout development with or without MPTP inhibition.

Ventricular cardiomyocytes were isolated enzymatically from $14(n=4), 28$ day old $(n=3)$ and adult rat heart $(n=3)$. These cardiomyocytes were incubated with $0.5 \mathrm{mM} \mathrm{H} 2 \mathrm{O} 2$ and $3 \mathrm{mM} \mathrm{Ca} 2+$ (simulated reperfusion) in the presence or absence of $2 \mu \mathrm{M}$ Cyclosporine A (CsA). Cardiomyocytes incubated in normal buffer were used as control. Cardiomyocyte viability (assessed using trypan blue) and morphology was monitored every $30 \mathrm{~min}$ for 2 hours.

There was a time-dependent decrease in viability in all age groups. However, this effect was more marked in 14 day old compared to adult and 28 day old cardiomyocytes. In the adult group, CsA significantly improved cardiomyocyte viability at all time points (at $120 \mathrm{~min}, 76 \% \pm 7.4$ vs $53 \% \pm 5.3$ ). No protective effect was seen in $14(22 \% \pm 12.7$ for CsA vs $10 \%$ \pm 6.6 injured control) or 28 day old cardiomyocytes $(55 \% \pm 7.9$ for CsA vs $33 \pm 5.4$ for injured control).

Unlike intact adult heart, the resistance of isolated cardiomyocytes to simulated reperfusion injury increases from 14 days of age to adulthood. The finding that CsA does not confer protection in the younger age group could be due to excessive injury or due to changes in MPTP sensitivity.

\section{P16 HYPERPOLARIZED 13C MAGNETIC RESONANCE SPECTROSCOPY IDENTIFIES CHANGES TO MYOCARDIAL METABOLIC FLUXES IN A RAT MODEL OF DOXORUBICIN-INDUCED CARDIOTOXICITY}

KN Timm, JJ Miller, V Ball, JA Henry, D Savic, MS Dodd, DJ Tyler. Department of Physiology Anatomy and Genetics, University of Oxford

10.1136/heartjnl-2018-BSCR.21

Doxorubicin (DOX) is a widely used anthracycline chemotherapeutic for adult and paediatric patients and its use has greatly improved cancer survival rates. However, DOX can cause serious cardiac side effects leading to congestive heart failure. The mechanisms for this toxicity are not yet fully understood, although mitochondrial oxidative stress and altered cardiac energetics are thought to play a key role in the pathology. In this study, we measured real-time metabolic fluxes in the rat heart following DOX treatment using hyperpolarized 13C magnetic resonance spectroscopy (MRS).

Rats were treated i.v. weekly for 6 weeks with either $2 \mathrm{mg} /$ $\mathrm{kg} \operatorname{DOX}(n=12$, low-dose) or saline $(n=12)$, or for 5 weeks with $3 \mathrm{mg} / \mathrm{kg}$ DOX $(n=8$, high-dose) or saline $(n=8)$. CINE MR imaging for cardiac functional analysis and hyperpolarized [1-13C]- and [2-13C]pyruvate MRS were performed at weeks 1,3 and 6.

DOX treatment lead to a progressive and dose-dependent decrease in cardiac ejection fraction and cardiac output. Those functional changes were accompanied by reduced pyruvate dehydrogenase flux in the high-dose model and reduced $13 \mathrm{C}$ label incorporation into the glutamate and acetyl-carnitine pool in both models, suggesting altered citric acid cycle flux and reduced acetyl-CoA buffering capacity in the myocardium. Rats showed variability in cardiotoxic severity and the metabolic and functional changes were significantly correlated.

Hyperpolarized 13C MRS is therefore a unique non-invasive method to reveal early metabolic effects of DOX on the heart. Future research will focus on unravelling the 